\title{
Impact of Data Presentation on Physician Performance Utilizing Artificial Intelligence-Based Computer-Aided Diagnosis and Decision Support Systems
}

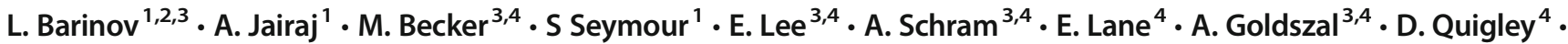 \\ L. Paster ${ }^{3,4}$
}

Published online: 15 October 2018

(C) The Author(s) 2018

\begin{abstract}
Ultrasound (US) is a valuable imaging modality used to detect primary breast malignancy. However, radiologists have a limited ability to distinguish between benign and malignant lesions on US, leading to false-positive and false-negative results, which limit the positive predictive value of lesions sent for biopsy (PPV3) and specificity. A recent study demonstrated that incorporating an AI-based decision support (DS) system into US image analysis could help improve US diagnostic performance. While the DS system is promising, its efficacy in terms of its impact also needs to be measured when integrated into existing clinical workflows. The current study evaluates workflow schemas for DS integration and its impact on diagnostic accuracy. The impact on two different reading methodologies, sequential and independent, was assessed. This study demonstrates significant accuracy differences between the two workflow schemas as measured by area under the receiver operating curve (AUC), as well as interoperator variability differences as measured by Kendall's tau-b. This evaluation has practical implications on the utilization of such technologies in diagnostic environments as compared to previous studies.
\end{abstract}

Keywords Breast cancer · Machine learning · Artificial intelligence · Clinical workflow · Computer-aided diagnosis (CAD) . Decision support

\section{Introduction}

Excluding skin cancer, breast cancer has the highest incidence rate and the second highest mortality rate in women [1]. Early and accurate diagnosis is a cornerstone strategy used to minimize breast malignancy, morbidity, and mortality. Imaging plays a central role in diagnosis; specifically, digital mammography/ tomosynthesis and ultrasound are the most frequently used screening and diagnostic modalities. In current imaging protocols, ultrasound (US) is a valuable tool for evaluating breast tissue, achieving sensitivity comparable to digital mammography (DM) and improved detection of invasive and node-negative

\footnotetext{
L. Barinov

lbarinov@princeton.edu

Koios Medical, New York, NY, USA

Princeton University, Princeton, NJ, USA

3 Rutgers University Robert Wood Johnson Medical School, New Brunswick, NJ, USA

4 University Radiology Group, East Brunswick, NJ, USA
}

breast cancers [2]. This improvement, however, comes at the cost of lower PPV3 and specificity [3]. In practice, the increased falsepositive rate manifests as an increase in benign biopsies.

One avenue being explored to address these concerns is the introduction of machine learning-based artificial intelligence (AI) systems. While these systems have been utilized in the past for mammography, their benefits have been recently called into question [4]. Additionally, rather than aiding in diagnosis, these systems have traditionally been used as an aid for the detection of suspicious areas [5-7]. This approach has been replicated in automated whole breast ultrasound (ABUS) but is only cleared to target areas not known to have suspicious findings [8].

More recently, tools have been developed to aid the diagnostic performance of radiologists, offering automated assessments of lesion characteristics and risk. Initial iterations have demonstrated a meaningful increase in sensitivity but a large decrease in specificity [4]. As machine learning techniques have progressed over the last 6 years, however, advances in performance within the diagnostic ultrasound space have followed suit.

A recent study demonstrated that integration of US with a new AI-based decision support (DS) system offers substantial improvement in both sensitivity and specificity. When tested 
alone, the DS platform was shown to exceed radiologist performance in US data analysis, showing a $34-55 \%$ potential reduction in benign biopsies and an increase in the positive predictive value of lesions sent for biopsy (PPV3) of 7-20\% [9].

While the system's raw performance is promising, DS's practical efficacy and impact also need to be assessed when integrated into existing real-world clinical workflows. This study investigates the clinical impact of two different diagnostic workflows. Clinical impact is evaluated as a function of how diagnostic support is presented. Presentation can either be sequential, where the clinician has an initial opportunity to evaluate the case unaided before receiving DS, or independent, where the case and decision support are presented together. Stand-alone clinician accuracy is compared to that of a clinician utilizing DS, and the system's impact on intraoperator and inter-operator variability is evaluated. The goal of this study is to evaluate workflow schemas for DS integration and their effects on diagnostic accuracy.

\section{Methods}

\section{Data Collection}

Using data acquired from the ACRIN 6666 trial [10], 500 cases were identified for inclusion. Lesion population statistics can be seen in Fig. 1. The dataset was enriched for malignancy, while all other statistics were chosen to approximate current population numbers per the Breast Cancer Research Foundation (BCRF) [10]. All pathological ground truth for malignant lesions came from biopsy-proven pathological follow-up, while for benign lesions, ground truth was established via biopsy or 1 year follow-up if the lesions were BI-RADS 4 and above or BI-RADS 3 and below, respectively. This dataset includes images obtained using a diverse set of US equipment and a range of high-frequency breast transducers (Fig. 2). Overall, this equipment and the lesion evaluated in the dataset accurately represent current clinical practice including inclusion of cases from both academic and nonacademic sites as well as dedicated breast and non-dedicated imaging centers [10].

\section{Machine Learning}

Utilizing original radiologist-designated regions of interest (ROI), two orthogonal views of each lesion were used to generate a machine learning system-based score [9]. These ROIs were selected by the radiologist who had originally read the case clinically. The score ranges from 0.0 to 1.0 and is subdivided into four categories, each representing a clinical course of action (Table 1).

These scores were presented to all study readers in a graphical form in the electronic Case Report Form (eCRF) (Fig. 3). a

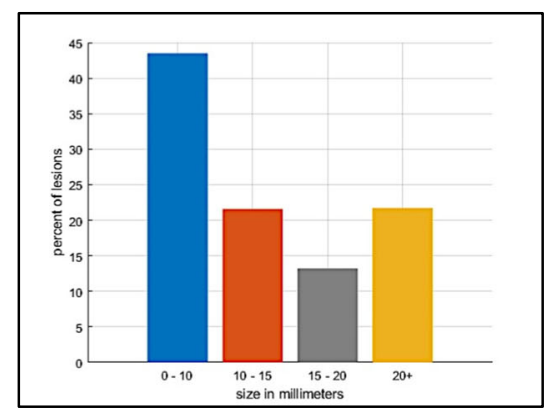

d

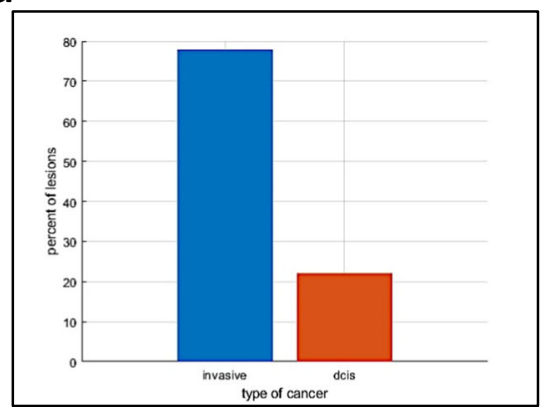

b

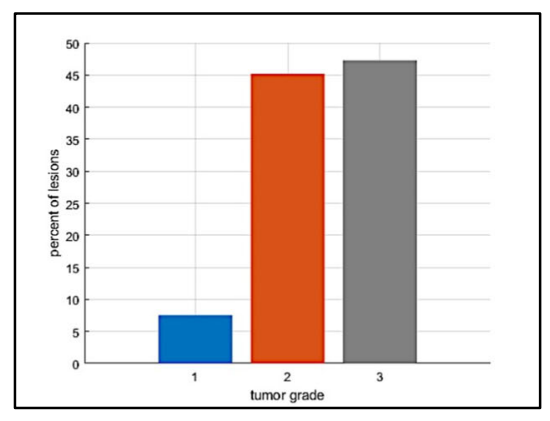

e

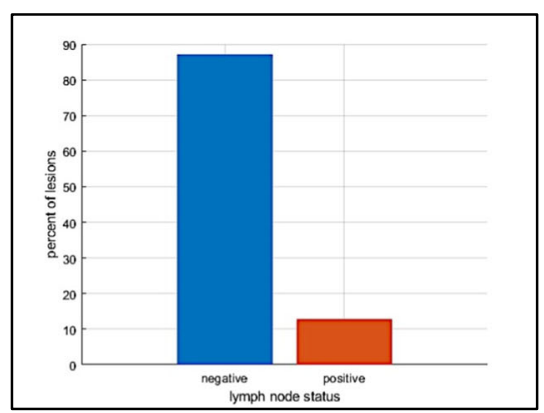

C

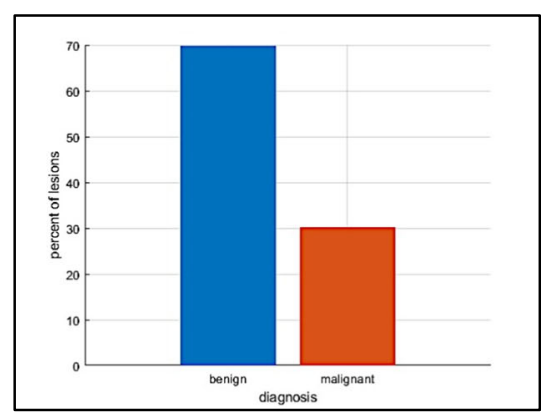

f

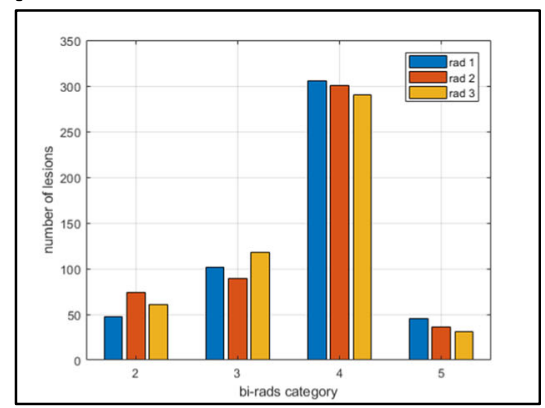

Fig. 1 Lesion population statistics. a Tumor size. b Tumor grade. $\mathbf{c}$ Benign or malignant. d DCIS (non-invasive) vs invasive. e Lymph node status. f BIRADS designation for the three radiologists tested 
Fig. 2 Ultrasound equipment characteristics. a Manufacturer. b Transducer frequency. " $\mathrm{n} / \mathrm{a}$ " refers to cases in which US transducer frequency was not recorded

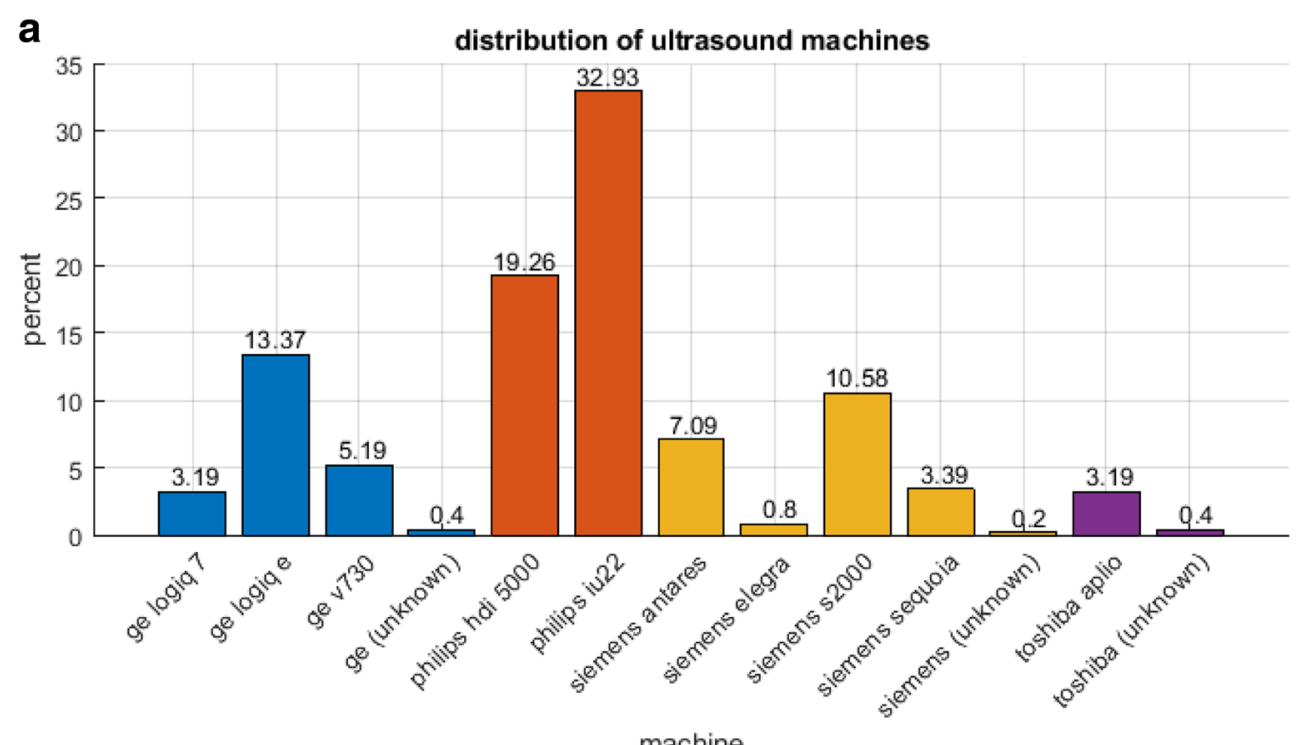

machine

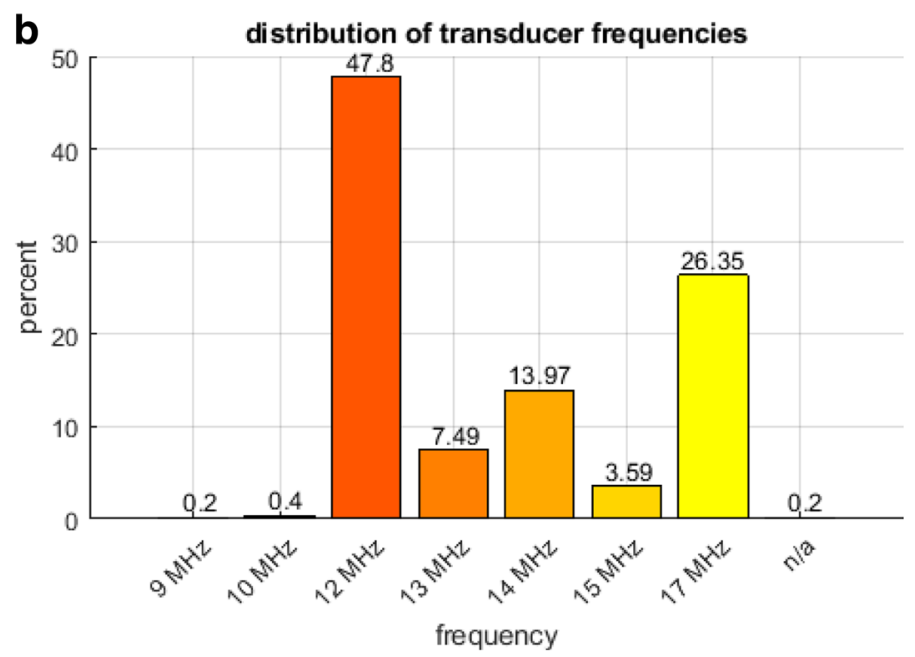

\section{Reader Characteristics and Training}

Three American Board of Radiology (ABR) certified radiologists, training and experience summarized in Table 2, evaluated the 500 case dataset. Each radiologist was initially given a 30-min training session to understand the machine learning output, stand-alone performance of the system, and the eCRF form. After training, each radiologist demonstrated proper utilization of the study platform by assessing 10 test cases proctored by the training staff.

Table 1 Score ranges and their corresponding categorical outputs. These ranges and categories are inherent to the system and were not designed or altered for this study

\begin{tabular}{ll}
\hline Categorical output & Score range \\
\hline Benign & {$[0,0.25)$} \\
Probably benign & {$[0.25,0.5)$} \\
Suspicious & {$[0.5,0.75)$} \\
Malignant & {$[0.751 .0]$} \\
\hline
\end{tabular}

Each reader specializes in breast imaging and performs a mix of screening and diagnostic breast imaging across multiple modalities.

\section{Reader Workflow}

Cases were presented to and scored by radiologists in a single software environment that showed orthogonal images, ROIs, and the DS output. The study platform then queried the radiologist to input a Breast Imaging Reporting and Data System (BI-RADS) score (the current clinical system used to evaluate lesion suspicion) and likelihood of malignancy (LoM) as a percentage (Fig. 3).

Using this software, the readers reviewed ultrasound images using two separate workflows which are summarized in Fig. 4:

\section{Sequential workflow}

1. Readers reviewed the case without the DS output (control read) 


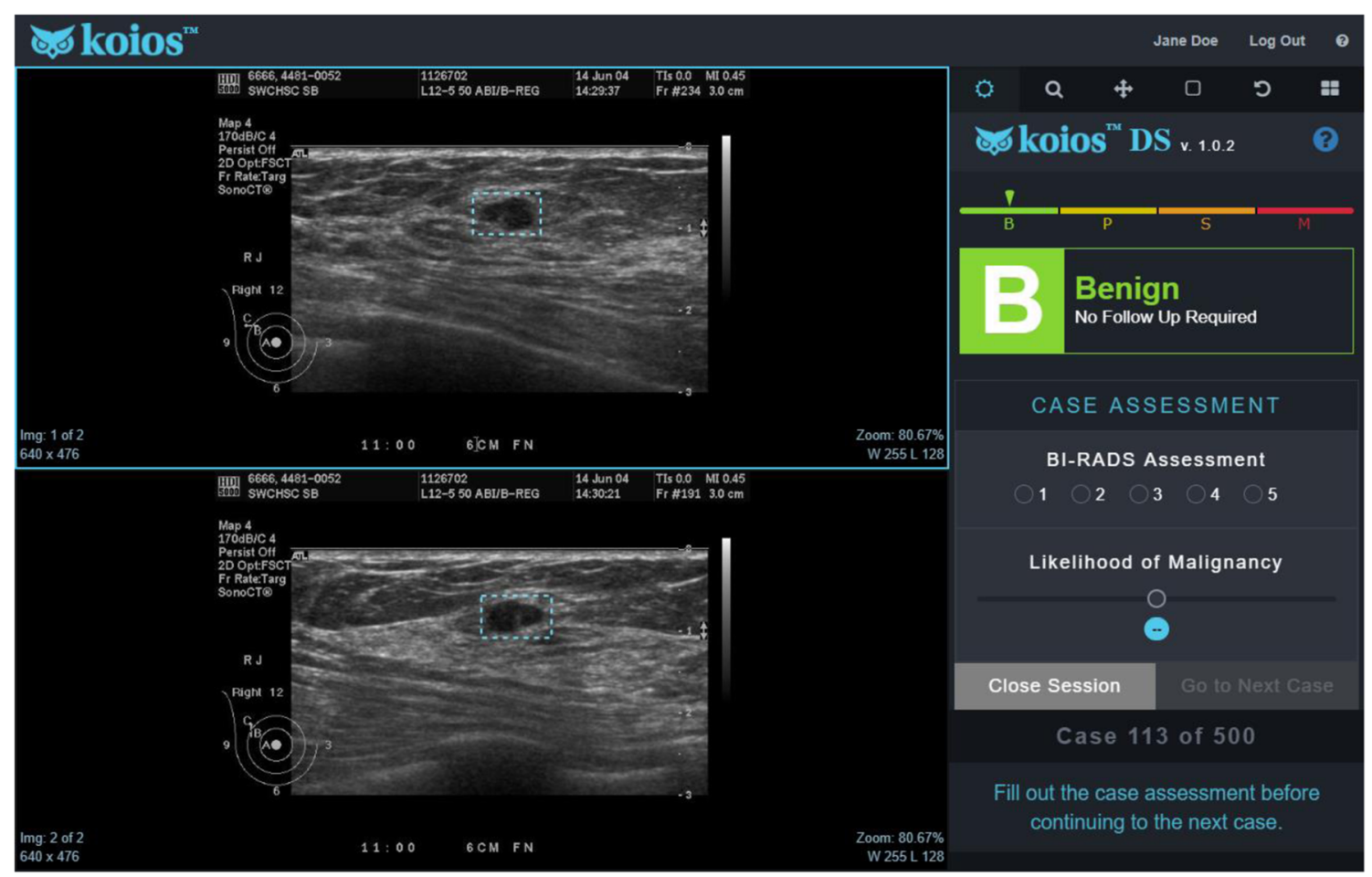

Fig. 3 Screen capture of the study platform. The left side shows two orthogonal views with ROIs. On the right side is the DS output and the radiologist case assessment input (BI-RADS assessment and likelihood of malignancy percentage)

2. Readers scored the case via BI-RADS and LoM

3. DS output was presented to the readers

4. Readers re-scored the case via BI-RADS and LoM

\section{Independent Workflow}

This workflow was employed after a 4-week "washout" period that followed the sequential workflow. After this washout period, readers where shown the images and the DS output, and they scored the case. In the independent workflow, readers were presented with 450 cases in randomized order. Fifty cases were withheld and reserved as control cases in order to measure intra-reader variability after the 4-week washout period. This workflow is summarized in the following sequence:

Readers were presented with one of the following:

1. Control case read with no DS output (50 Total)

Table 2 This table provides a summary of the three readers involved in this study

\begin{tabular}{llll}
\hline Radiologist ID & $\begin{array}{l}\text { Post-educational training } \\
\text { experience (years) }\end{array}$ & $\begin{array}{l}\text { ABR } \\
\text { certified }\end{array}$ & $\begin{array}{l}\text { Breast fellowship } \\
\text { training }\end{array}$ \\
\hline 1 & $20+$ & $\mathrm{x}$ & $\mathrm{x}$ \\
2 & $10+$ & $\mathrm{x}$ & $\mathrm{x}$ \\
3 & $5+$ & $\mathrm{x}$ & \\
\hline
\end{tabular}

(a) Readers scored the case via BI-RADS and LoM OR

2. A case containing DS output (450 Total)

(b) Readers scored the case via BI-RADS and LoM

\section{System Evaluation}

Radiologists were presented with DS output that used the original ROIs selected during clinical evaluation of the cases. This choice was made to enhance reader efficiency so that a high volume of cases could be evaluated in a practical time frame. However, while it is felt that the ROI a radiologist chooses manually would be very similar to the ROI used in this study, it is possible that results would be impacted the variation caused by manually demarcated ROIs. To evaluate the system's robustness to variation in the ROI boundaries, two assessments were performed.

First, DS output's robustness to ROI boundary variation was assessed by evaluating the 500 cases 20 times, randomly varying the ROI boundary each time. Specifically, each corner of the region was shifted at random by up to $20 \%$ in each dimension from the predetermined optimal cropping. ROC curves and the AUC distributions were calculated.

Second, the boundaries between BI-RADS 3 Probably Benign (P) and BI-RADS 4a Suspicious (S) represents the most clinically impactful diagnostic decision point. It is 

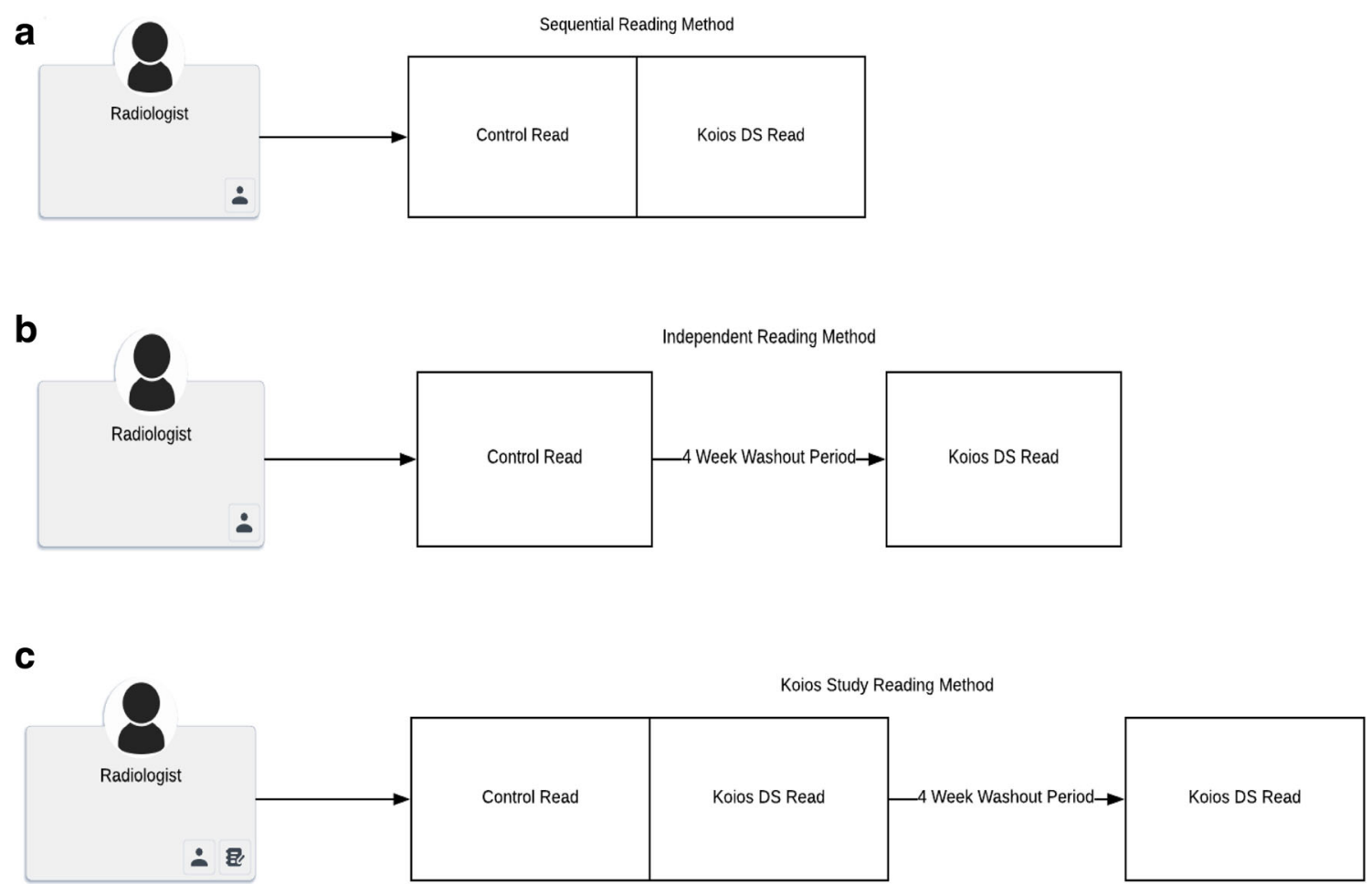

Fig. 4 Schematic representation of the $\mathbf{a}$ sequential and $\mathbf{b}$ independent reading paradigms. A combination approach seen in $\mathbf{c}$ was utilized in this study

critical to understand the effects of ROI variability on class switching across categories, and specifically the P-S boundary. Since the system's output is categorical, changes across this decision boundary have the potential to change clinical management. The level of class switching due to ROI sensitivity was evaluated by utilizing the 20 ROIs generated in the previous analysis and counting the number of times category switching is observed compared to the initial radiologist supplied cropping.

Finally, in order to verify the relationship between the system's categorical output and BI-RADS, a sensitivity and specificity analysis was conducted. For each categorical group, the sensitivity and specificity values were compared between the system and each participating reader's BI-RADS assessment.

\section{Reader Evaluation}

The area under the receiver operating curve (AUC) for each radiologist across each reading paradigm was calculated and compared to their control reads. The estimate of the change in AUC as well as the $95 \%$ confidence intervals was made by using the Dorfman-Berbaum-Metz method of MRMC analysis using the Metz-ROC LABMRMC software [11].

Intra- and inter-operator variability was assessed via Kendall's tau-b correlation coefficient [12]. This assessment was done in a pairwise fashion across each pair of readers before and after being provided with the decision support output and across each reading methodology.

\section{Results}

System Evaluation: Variability in ROI boundary produces no significant change in either the shape of the ROC curve or the AUC values (Fig. 5a). Similarly, the ROI analysis shows minimal class switching between $\mathrm{P}-\mathrm{S} / \mathrm{S}-\mathrm{P}$ categories, 2.7 and $3.3 \%$, respectively (Fig. 5b). We further correlate the results for each of the categorical groups supplied by the system to the BI-RADS assessments provided by the radiologist readers (Fig. 6).

Analysis of the operating points of the system appear to be as good, or better, than the radiologists tested in this study (Fig. 6). This suggests that the performance of categorical outputs of the system align to and exceed the performance of the BI-RADS assessments.

The reader evaluation analysis showed a system only AUC [95\% confidence interval (CI)] of 0.8648 [0.8345-0.8893]. Each radiologist's stand-alone performance is detailed in Table 3.

Similarly, the sequential and independent joint performance is summarized in Table 4 and Fig. 7.

Intra-reader and inter-reader variability, as measured by Kendall's tau-b, is summarized in Tables 5 and 6, respectively.

\section{Discussion}

The current study confirms that this DS system performs favorably when compared with a radiologist's performance, 
a

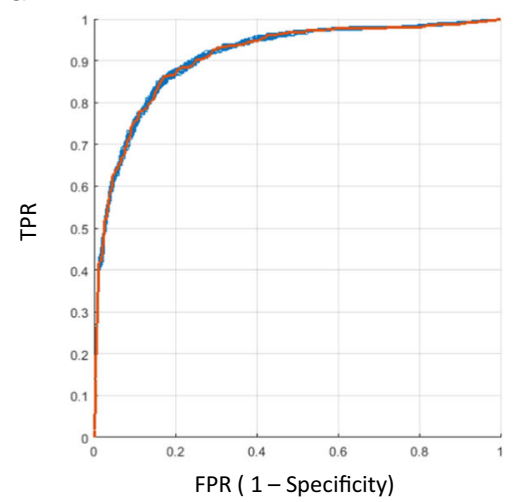

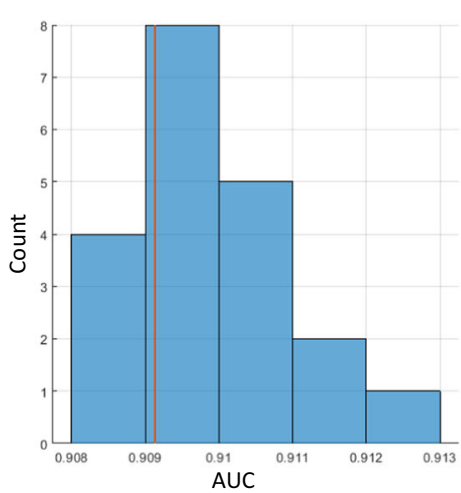

b

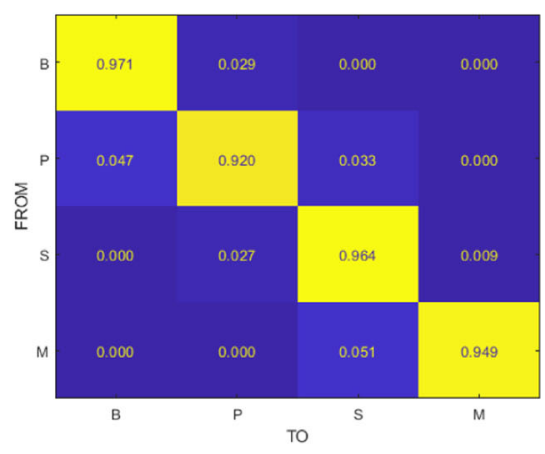

Fig. 5 Results of the system evaluation. a ROC curves and corresponding AUCS assessing impact of ROI boundary variation. b Assessment of class switching due to ROI boundary variation

confirming prior studies [9]. In addition, rather than simply comparing DS system performance to that of a radiologist, it extends these prior results by assessing workflows that more realistically approximate clinical practice. Past studies have shown mixed evidence on the effect of the tested reading methodologies on overall reader performance, but none have conducted an investigation within the context of diagnostic decision support, and their effects remain unknown [13]. Our results show a sizeable variation in performance obtained depending on which reading methodology was chosen (Table 4). The impact of the reading methodology on study performance has practical workflow considerations. These results suggest there may a strong impact of confirmation bias in a sequential study design. This can be clearly seen in Fig. $7 \mathrm{~b}$ versus Fig. 7c, where the deviation from the control assessment is significantly smaller in the sequential read versus independent read. This has practical implications in the utilization of machine learning decision support in breast ultrasound diagnostics and likely beyond.

Fig. 6 Sensitivity and specificity of each reader's BI-RADS grading is compared to that of the systems corresponding categorical output
Furthermore, as would be expected by providing a supplemental, concurrent read that out-performs the original reader, overall inter-operator variability decreased significantly. Surprisingly, inter-operator variability decreased even beyond that of the 4-week washout intra-reader variability per Tables 5 and 6 . Due to the study design, the effects of a machine learning-based concurrent read on intra-operator variability were not evaluated, but with the evidence presented in this study, it would seem likely that a proportional decrease in this metric could be expected.

\section{Clinical Applications}

When looking at practical clinical workflow applications, the performance results and study design have a number of implications on the application of AI software.

In clinical practice, the typical sequence of radiology workflow is:

\section{Sensitivity}

\begin{tabular}{|c|c|c|c|c|}
\hline System & $1.00[0.971 .00]$ & $0.98[0.951 .00]$ & $0.95[0.890 .98]$ & $0.50[0.410 .59]$ \\
\hline & B & $\mathrm{P}$ & s & M \\
\hline rad-1 & $1.00\left[\begin{array}{lll}0.97 & 1.00\end{array}\right]$ & $0.96\left[\begin{array}{ll}0.91 & 0.99\end{array}\right]$ & $0.92\left[\begin{array}{lll}0.87 & 0.96\end{array}\right]$ & $0.19\left[\begin{array}{lll}0.13 & 0.27\end{array}\right]$ \\
\hline rad-2 & $1.00\left[\begin{array}{lll}0.97 & 1.00\end{array}\right]$ & $0.99\left[\begin{array}{lll}0.96 & 1.00\end{array}\right]$ & $0.95\left[\begin{array}{lll}0.90 & 0.98\end{array}\right]$ & $0.23\left[\begin{array}{ll}0.17 & 0.32\end{array}\right]$ \\
\hline rad-3 & $1.00\left[\begin{array}{lll}0.97 & 1.00\end{array}\right]$ & $1.00\left[\begin{array}{lll}0.97 & 1.00\end{array}\right]$ & $0.97[0.920 .99]$ & $0.29\left[\begin{array}{lll}0.21 & 0.37\end{array}\right]$ \\
\hline
\end{tabular}

Specificity

\begin{tabular}{|c|c|c|c|c|}
\hline System & $0.43[0.380 .48]$ & $0.62[0.57 \quad 0.67]$ & $0.96\left[\begin{array}{lll}0.94 & 0.98\end{array}\right]$ & $1.00[0.991 .00]$ \\
\hline
\end{tabular}

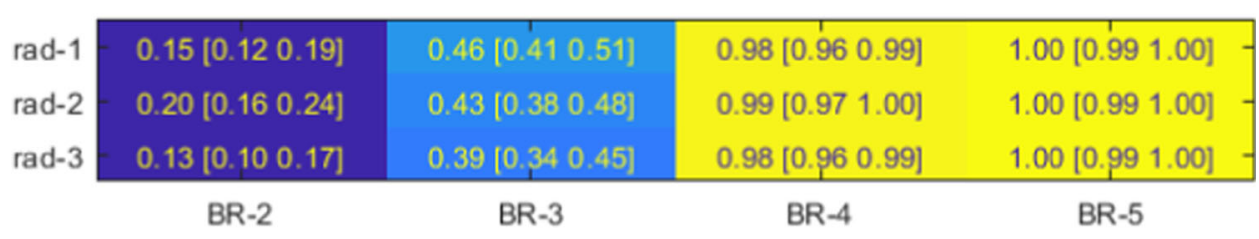


Table 3 Each reader's performance was assessed prior to being presented the system's output. The results of their control reads as measured via AUC is shown in this table

\begin{tabular}{ll}
\hline Radiologist ID & AUC, 95\% CI \\
\hline 1 & $0.7618[0.7244-0.7934]$ \\
2 & $0.7543[0.7197-0.7887]$ \\
3 & $0.7325[0.6897-0.7689]$ \\
\hline
\end{tabular}

1. Radiologist assesses mammographic images and instructs technologist to perform a complete mammographic examination

2. Radiologist decides if US is needed

3. If so, the technologist acquires images and presents to radiologist

4. Radiologist assesses images and the radiologist may or may not confirm the results with radiologist real time scanning

5. Radiologist formulates diagnosis

In the sequential workflow, the radiologist would complete "step 5" then assess the DS output. In the independent workflow, the DS output would be presented to the radiologist during "step 4," along with the US images (e.g., along with the other US “data").

In clinical practice, if the radiologist has confidence in the DS system, the independent workflow seems more likely to impact clinical management, e.g., the radiologist looks at all the data (demographic/clinical history, mammographic, US, DS output) and forms a diagnosis (the ultimate goal).

\section{Comparison to Other Systems}

Prior research has explored the difference between sequential and independent study designs and their respective effects on performance [13-16]. These studies have suggested that both designs produce similar performance results within comparative analyses. They then conclude that sequential designs are preferable due to lower time and resource requirements.
Interestingly, our results seem to suggest a more significant deviation between these two study designs.

The difference between our results and the results discussed above can perhaps be attributed to the following factors. The first consideration which must be made is that the technology being tested and the underlying modality are both different. Second, the task being performed by the decision support system is all-together different from the CAD systems being examined in these studies. Most of the systems under study are focused on detection, while DS is focused on diagnosis.

In detection, a sequential read implies that the clinician identifies regions of interest, performs CAD, and then potentially examines additional regions, as suggested by the CAD device. When performing an independent read, that clinician will see regions of interest as identified by $\mathrm{CAD}$ and may then examine additional regions based upon their own inspection. In both cases, the clinician is combining their detection results with the CAD's, so it is reasonable that performance is similar between the two.

In diagnosis, a sequential read implies that the clinician will examine a nodule, arrive at a conclusion, and then receive the recommendation of the DS system. The recommendation will either confirm their decision, alter their decision, or not be considered. In an independent read, the recommendation is presented at the outset, and is considered as additional input data alongside the image as the clinician makes a decision. In the sequential case, since the clinician has already formed an opinion, a dissenting recommendation may meet with intransigence, and wind up suffering from confirmation bias.

\section{Limitations}

While the results suggest a strong performance benefit, there are several limitations to the study design that must be taken into consideration. The study only consisted of three (3) readers. Although the readers had varying degrees of experience within the study, the study does not capture the full breadth of readers across the broader population of radiologists that read and interpret breast ultrasound images. The
Table 4 In order to compare the two reading methodologies, the readers' performance was assessed via AUC compared to their control reads summarized in Table 3 . None of the readers attained statistical significance when utilizing sequential reads, while all readers were significantly better when utilizing an independent reader strategy

\begin{tabular}{|c|c|c|c|c|}
\hline Radiologist ID & $\begin{array}{l}\text { Sequential read } \\
\text { AUC, } 95 \% \text { CI }\end{array}$ & $\begin{array}{l}P \text { value CR vs } \mathrm{SR} \\
\text { two-tailed alpha }=0.05\end{array}$ & $\begin{array}{l}\text { Independent read A } \\
\text { UC, 95\% CI }\end{array}$ & $\begin{array}{l}P \text { value CR vs IR } \\
\text { two-tailed alpha }=0.05\end{array}$ \\
\hline 1 & $0.7935[0.7567-0.8229]$ & 0.235 & $\begin{array}{l}0.8213 \\
{[0.7861-0.8516]}\end{array}$ & $0.0285^{*}$ \\
\hline 2 & $0.7674[0.7327-0.8001]$ & 0.601 & $\begin{array}{l}0.8305 \\
{[0.7982-0.8594]}\end{array}$ & $0.00155^{*}$ \\
\hline 3 & $0.7859[0.7527-0.8174]$ & 0.0532 & $\begin{array}{l}0.7988 \\
{[0.7632-0.8310]}\end{array}$ & $0.0160^{*}$ \\
\hline
\end{tabular}

\footnotetext{
*Significant
} 
a

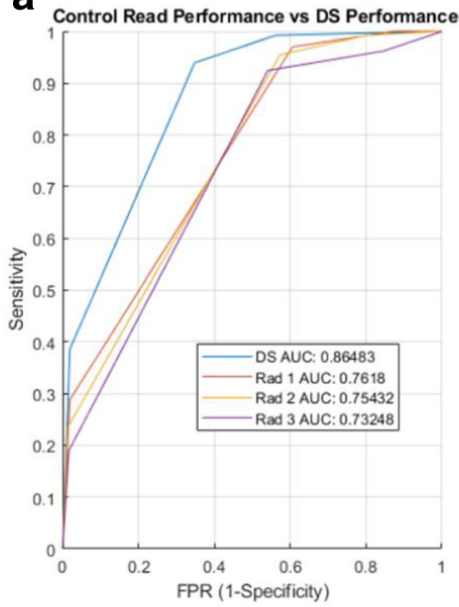

b

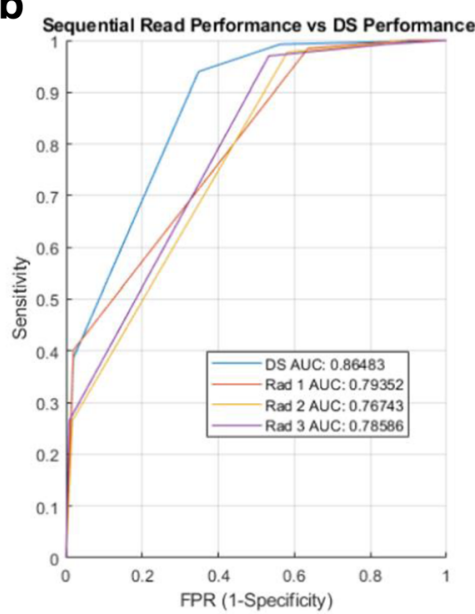

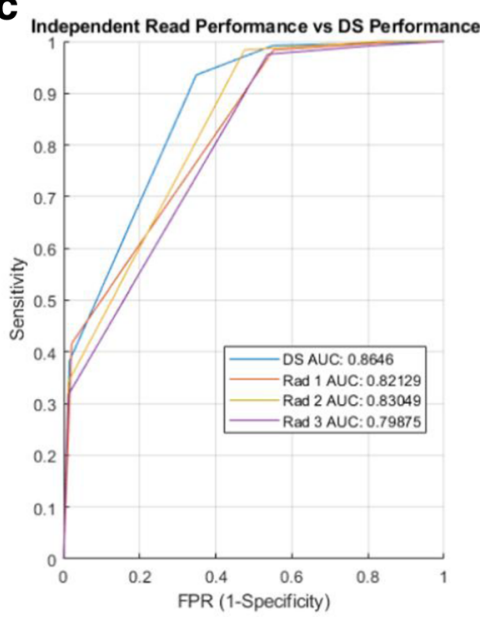

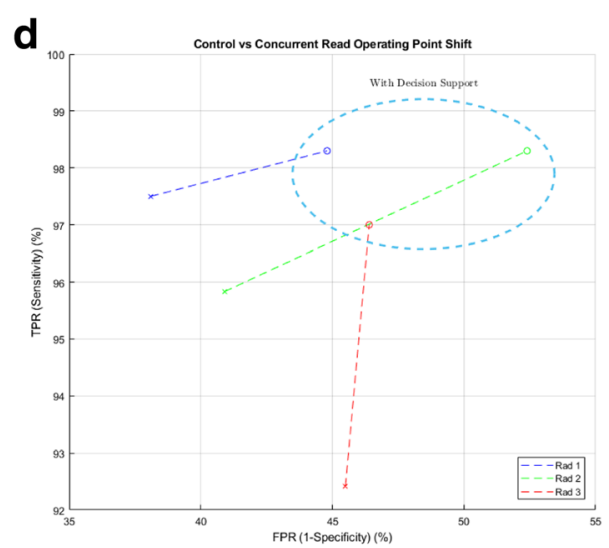

Fig. 7 Comparative assessment of a control, $\mathbf{b}$ sequential, and $\mathbf{c}$ independent reading workflows. Operating point specific improvement for Independent vs control assessments were additionally measured (D)

number of cases (500) and enrichment within the study may also limit its ability to represent the typical distribution of cases that a reader would expect to see in practice. The choice of distribution of the cases was an attempt to create a set that was broadly representative of the greater populations of lesions as a whole but could also answer questions in a statistically meaningful way about events that have low population incidence. This further extends to the retrospective nature of the study design. In clinical practice, additional factors impact clinical reasoning that are not fully represented in the study, such as patient history, prior studies, and patient preference towards clinical follow up. While a prospective, randomized

Table 5 To further characterize reader performance, intra-reader variability was measured via Kendall's tau-b

Radiologist ID

Kendall's tau-b for intra-reader variability assessment.
0.597
0.595
0.529

control study would have addressed some of these concerns, it would come at the cost of study time and complexity.

This study did not compare intra-operator variability with and without a decision support output as it would have required an additional set of reads for each participant. Based on the current results, it would seem likely that the intra-operator variability would decrease, but without study modification, the occurrence and extent of the variability is unknown.

The first reading session of this study was not counterbalanced, and all readers initially read sequential first and independent second. Cases without a corresponding DS output were randomly introduced in the independent session to break up the reading schedule and allow for the evaluation of intra-operator variability. The lack of counterbalancing between sequential and independent reads may have introduced slight reader bias when comparing the two paradigms.

Finally, the current study assesses the impact of DS on US interpretation in isolation, when in fact a complete breast imaging assessment incorporates demographic data, clinical history, and other imaging modalities such as mammography. New and exciting avenues of inquiry are needed to more fully evaluate the role and utility of DS needs in this larger context. 
Table 6 Pairwise combinations of variability were measured utilizing the sequential read (SR) methodology. Interestingly, the independent read (IR) variability was lower than intra-reader variability

\begin{tabular}{llll}
\hline $\begin{array}{l}\text { Radiologist ID (KTB } \\
\text { for reading methods) }\end{array}$ & 1 (control, SR, IR) & 2 (control, SR,IR) & 3 (control, SR,IR) \\
\hline 1 (control, SR,IR) & $(1,1,1)$ & $\left(0.5505,0.6263^{*}, 0.7231^{* *}\right)$ & $\left(0.4944,0.6640^{*}, 0.7476^{* *}\right)$ \\
2 (control, SR,IR) & - & $(1,1,1)$ & $\left(0.4229,0.5641^{*}, 0.6231^{* *}\right)$ \\
3 (control, SR,IR) & - & - & $(1,1,1)$ \\
\hline
\end{tabular}

*Significant with $p<.01 ; * *$ significant difference with $p<1 \mathrm{e}-8$

\section{Conclusion}

We have been able to demonstrate that reader workflow can significantly affect clinical performance when incorporating AI-based decision support tools. This evaluation has novel practical implications on the utilization of such technologies in diagnostic environments as compared to previous studies which have concluded an effective equivalence between these two reading paradigms. Independent reads (concurrent reads) have shown dramatic shifts in reader performance and interoperator variability as compared to either control reads or sequential reads. The evidence provided in this study can be used to impact both study design when demonstrating efficacy of new diagnostic decision support tools, as well as their implementation in practical environments.

Acknowledgements We would like to thank Dr. Bruce McClennan, William Hulbert, and Graham Anderson for all of the discussion and insight provided to make this work possible.

Open Access This article is distributed under the terms of the Creative Commons Attribution 4.0 International License (http:// creativecommons.org/licenses/by/4.0/), which permits unrestricted use, distribution, and reproduction in any medium, provided you give appropriate credit to the original author(s) and the source, provide a link to the Creative Commons license, and indicate if changes were made.

\section{References}

1. Siegel RL, Miller KD, Jemal A: Cancer statistics, 2018. CA: A Cancer Journal for Clinicians 68:7-30, 2018. https://doi.org/10. 3322/caac. 21442

2. Bae MS, Han W, Koo HR, Cho N, Chang JM, Yi A, Park I, Noh D, Choi WS, Moon WK: Characteristics of breast cancers detected by ultrasound screening in women with negative mammograms. Cancer Science 102:1862-1867, 2011. https://doi.org/10.1111/j. 1349-7006.2011.02034.x

3. Berg, Wendie A., Bandos, Andriy I., Mendelson, Ellen B., Lehrer, Daniel, Jong, Roberta A., Pisano, Etta D.; Ultrasound as the primary screening test for breast cancer: analysis from ACRIN 6666, JNCI: Journal of the National Cancer Institute, Volume 108, Issue 4, 1 April 2016, djv367, https://doi.org/10.1093/jnci/djv367

4. Chabi M, Borget I, Ardiles R, Aboud G, Boussouar S, Vilar VS, Dromain C, Balleyguier C: Evaluation of the accuracy of a computeraided diagnosis (CAD) system in breast ultrasound according to the radiologist's experience. Academic radiology 19(3):311-319, 2012

5. Gur, D., Sumkin, J.H., Rockette, H.E., Ganott, M., Hakim, C., Hardesty, L., Poller, W.R., Shah, R., Wallace, L.; Changes in breast cancer detection and mammography recall rates after the introduction of a computer-aided detection system, JNCI: Journal of the National Cancer Institute, Volume 96, Issue 3, 4 February 2004, Pages 185-190, https://doi.org/10.1093/jnci/djh067.

6. Litjens G, Debats O, Barentsz J, Karssemeijer N, Huisman H: Computer-aided detection of prostate cancer in MRI. in IEEE Transactions on Medical Imaging 33(5):1083-1092, 2014. https:// doi.org/10.1109/TMI.2014.2303821 http://ieeexplore.ieee.org/ stamp/stamp.jsp?tp=\&arnumber=6729091\&isnumber $=6803900$

7. Van Ginneken B, Ter Haar Romeny BM, Viergever MA: Computer-aided diagnosis in chest radiography: a survey. in IEEE Transactions on Medical Imaging 20(12):1228-1241, 2001. https://doi.org/10.1109/42.974918 http://ieeexplore.ieee.org/ stamp/stamp.jsp?tp=\&arnumber=974918\&isnumber $=21028$

8. FDA.gov. QVIEW Medical, Inc. Premarket approval for QVCAD system. Summary of safety and effectiveness. Approved: November 9, 2016. https://www.accessdata.fda.gov/cdrh_docs/ pdf15/P150043B.pdf

9. Barinov L, Jairaj A, Paster L, Hulbert W, Mammone R, Podilchuk $\mathrm{C}$ : Decision quality support in diagnostic breast ultrasound through artificial intelligence. IEEE Signal Processing in Medicine and Biology Symposium (SPMB)., 2016. https://doi.org/10.1109/ spmb.2016.7846873

10. Data were obtained from the NCI-funded Breast Cancer Surveillance Consortium (HHSN261201100031C). Downloaded on [2018, February 21] from the Breast Cancer Surveillance Consortium website, http://breastscreening.cancer.gov/.

11. Dorfman DD, Berbaum KS, Metz CE: Receiver operating characteristic rating analysis: Generalization to the population of readers and patients with the jackknife method. Investigative Radiology. 27:723-731, 1992

12. Kendall M: A new measure of rank correlation. Biometrika. 30(1-2):8189, 1938. https://doi.org/10.1093/biomet/30.1-2.81.JSTOR2332226

13. Schalekamp S, van Ginneken B, Schaefer-Prokop CM, Karssemeijer N: Influence of study design in receiver operating characteristics studies: Sequential versus independent reading. Journal of Medical Imaging. 1(1):015501, 2014. https://doi.org/ 10.1117/1.JMI.1.1.015501 https://www.ncbi.nlm.nih.gov/pmc/ articles/PMC4478981/.

14. Beiden SV, Wagner RF, Doi K, Nishikawa RM, Freedman MB-L, Shih-Chung X, Xin-Wei: Independent versus sequential reading in ROC studies of computer-assist modalities. Academic Radiology. 9(9):1036-1043, 2002 https://www.sciencedirect.com/science/ article/pii/S1076633203804798

15. Gallas BD, Chan H-P, D’Orsi CJ, Dodd LE, Giger ML, Gur D, Krupinski EA, Metz CE, Myers KJ, Obuchowski NA, Sahiner B, Toledano AY, Zuley ML: Evaluating imaging and computer-aided detection and diagnosis devices at the FDA. Academic radiology. 19(4):463-477, 2012. https://doi.org/10.1016/j.acra.2011.12.016 https://www.ncbi.nlm.nih.gov/pmc/articles/PMC5557046/\#R73.

16. Obuchowski NA, Meziane M, Dachman AH, Lieber ML, Mazzone PJ: What's the control in studies measuring the effect of computeraided detection (CAD) on observer performance? Academic Radiology. 17:761-767, 2010 https://www.ncbi.nlm.nih.gov/ pubmed/20457419 